\title{
Inversion domains in AIN grown on (0001) sapphire
}

\author{
J. Jasinski ${ }^{1}$ and Z. Liliental-Weber \\ Lawrence Berkeley National Laboratory, Materials Science Division, 1 Cyclotron Rd., \\ Berkeley, CA 94720 \\ Q. S. Paduano and D. W. Weyburne \\ Air Force Research Laboratory, Sensors Directorate 80 Scott Dr., Hanscom AFB, MA \\ 01731
}

\begin{abstract}
Al-polarity inversion domains formed during AlN layer growth on (0001) sapphire were identified using transmission electron microscopy (TEM). They resemble columnar inversion domains reported for GaN films grown on (0001) sapphire. However, for AlN, these columns have a V-like shape with boundaries that deviate by $2 \pm 0.5^{\circ}$ from the c-axis. TEM identification of these defects agrees with the post-growth surface morphology as well as with the microstructure revealed by etching in hot aqueous $\mathrm{KOH}$.
\end{abstract}

\footnotetext{
${ }^{1}$ E-mail: jbjasinski@lbl.gov
} 
In recent years there has been an increasing interest in producing high quality AlN films. Being a compound semiconductor with a hexagonal wurtzite structure and a wide energy bandgap of $6.2 \mathrm{eV}$, AlN has many potential applications in optoelectronic devices, especially in UV emitters [1]. It has also a high breakdown voltage of $14 \mathrm{kV} / \mathrm{mm}$ and high thermal conductivity of $320 \mathrm{~W} /(\mathrm{mK})$, which makes it attractive for high power and high temperature applications [2]. Moreover, due to their high ultrasonic velocity of $6000 \mathrm{~m} / \mathrm{s}$, strong piezoelectricity, and good thermal stability, AlN thin films can be used for GHz-band surface acoustic wave devices [3].

Substrate technology remains an important issue for III-nitride device technology. Despite its large lattice mismatch with III-nitrides and its large differences in thermal expansion coefficient, c-plane sapphire has remained the substrate of choice for many applications. As one would expect, structural defects are a major material issue. Numerous studies of $\mathrm{GaN}$ films grown on sapphire have revealed a number of different structural defects. Threading dislocations seem to be the most dominant among them; however, other defects such as dislocation loops, basal stacking faults, nanopipes, and inversion domains (IDs) are also important and have a significant impact on the film quality and on the device performance. In the case of AlN films grown on sapphire one expects similar defects to be formed. To date, little has been done so far to identify and characterize these defects.

This paper reports a structural study of V-shape columnar defects formed inside AlN films grown on sapphire substrates. Several experimental results are provided to prove that these defects are, indeed, inversion domains. Similarities and differences between these IDs formed in AlN/sapphire and IDs observed in GaN/sapphire are also discussed 
AlN films were grown directly on a $50 \mathrm{~mm}$ diameter (0001)-oriented sapphire substrate by metal organic chemical vapor deposition (MOCVD) using an EMCORE DS75 reactor. The growth was initiated with substrate nitridation, performed under ammonia flow of $650 \mathrm{sccm}$ for $45 \mathrm{~s}$ at $930^{\circ} \mathrm{C}$. The temperature was then increased to $1150^{\circ} \mathrm{C}$, and the $\mathrm{AlN}$ layer, having a thickness of $\sim 500 \mathrm{~nm}$, was grown. Details of the growth procedure can be found elsewhere [4]. The AlN layer was investigated using atomic force microscopy (AFM) and transmission electron microscopy (TEM).

Two AFM images, the first showing the variation of the surface potential and the second showing the variation of surface height, obtained from the same area of the AlN layer, are shown in Figs. 1a and 1b, respectively. One can see from these images that there is a high density (of $\sim 3 \times 10^{9} \mathrm{~cm}^{-2}$ ) of small islands on the surface. Their average in-plane diameter is about $40 \mathrm{~nm}$, and their heights range from 30-40 nm. Many of these islands are located in the middle of much larger, six-fold faceted islands of the surrounding material.

The microstructure of the layer as revealed by cross-sectional TEM is shown in Fig. 1c. It can be noticed that the film contains a relatively high density of threading dislocations (of the order of $10^{10} \mathrm{~cm}^{-2}$ ). However, the more interesting defects visible in Fig. 1c are narrow columns having a characteristic V-like shape. Their estimated density is $\sim 5 \times 10^{9} \mathrm{~cm}^{-}$ ${ }^{2}$. All of them nucleated at the AlN/sapphire interface; their initial widths are $\sim 10 \mathrm{~nm}$ or less. During the growth these columns widen at a steady rate, resulting in a V-like shape with a semi-angle of $2 \pm 0.5^{\circ}$. Fig. 1c shows also that tips of these columns stick out from the layer surface, very often in the middle of islands of the surrounding material. These tips have heights of 30-40 nm; many have very well-defined facets, as shown in the inset of the Fig. $1 \mathrm{c}$. 
A comparison of AFM and TEM images shown in Figs 1a-1c leads directly to the conclusion that the small islands detected by AFM on the layer surface are simply the tips of V-like columns revealed by TEM. A discrepancy between defect counts provided by both methods may be due to the layer non-uniformity, or because cross-sectional TEM permits only a rough defect density estimation, at least as long as rather complex specimen thickness measurement procedures are not carried out.

In order to understand the nature of these columnar defects we performed more detailed diffraction contrast TEM experiments. These experiments reveal that the columnar defects are out of contrast for diffraction conditions where the g-vector is perpendicular to the c-axis (see Fig. 2a). On the other hand, boundaries of these columns show strong strain contrast in dark field images obtained with $\mathbf{g}=(0002)$ (see Fig. $2 b)$. These results indicate that there is a displacement associated with these boundaries along the c-axis. Thus, these columnar defects may be IDs since the same type of displacement vector has been reported for inversion domain boundaries (IDBs) in AIN [5] and GaN [6]. In the following we will prove that these defects are indeed IDs.

There are several methods to identify IDs. One of them, proposed by Serneels et al. [7], is based on the violation of Friedel's law in the case when multiple diffraction conditions along a noncentrosymmetric zone axis are applied. For a wurtzite structure one such zone axis is the [1100] zone axis, where there is no center of symmetry and the intensity distribution within the (0002) electron diffraction disk can not be obtained by simple symmetry operations of the intensity distribution within the (0002) disk. The asymmetry between the (0002) and the (0002) disks leads to the contrast difference between adjacent regions of opposite polarities in dark-field images recorded under these conditions 
with one of these two $\mathbf{g}$-vectors. Therefore, a pair of dark-field images, one with $\mathbf{g}=(000 \underline{2})$, and the other with $\mathbf{g}=(0002)$, will have complementary contrasts (dark areas will appear bright and vice versa). Due to a reversal in the Al-N bonding, the diffraction pattern of an inversion domain is reversed compared to that of the matrix, and the (0002) disk of the inversion domain has the same intensity distribution as the (0002) disk of the matrix.

Such pairs of dark-field images of AlN film on sapphire exhibiting complementary contrast are shown in Figs. 3a,b. Four columnar defects are visible in these images. In the first image three of them are darker than the matrix, whereas the same three defects are brighter than the matrix when the opposite diffraction beam is used. These experimental results prove that V-like columnar defects in our AlN film on sapphire are IDs.

The second method for revealing the inversion character of IDs is based on the convergent beam electron diffraction (CBED) technique. It allows for straightforward identification of regions of inverted polarity because the CBED pattern of a noncentrosymmetric zone axis recorded at the ID is reversed relative to that recorded at the adjacent matrix material. However, since CBED patterns are strongly thickness-dependent, then to use this method correctly one must make sure the TEM specimen thickness is the same in these two areas. We applied this method to V-like columnar defects observed in the AlN film choosing an [1100] zone axis orientation of the sample. Two CBED patterns, one recorded inside and one recorded outside such a defect are shown in Fig. 3c. The intensity distribution within the central (0000) disk is the same for both patterns, demonstrating that the specimen thickness in both areas is the same. On the other hand, when (0002) and (0002) disks are considered, a clear reversed intensity distribution is visible between the pattern recorded on the ID and the pattern recorded at the adjacent matrix material. This again 
proves that the V-like columnar defects are IDs. The overall asymmetry of CBED patterns in Fig. $3 \mathrm{c}$ is rather weak compared to the asymmetry of CBED [1100] patterns recorded for $\mathrm{GaN}$. This is due to the fact that the atomic number difference between $\mathrm{Al}$ and $\mathrm{N}$ atoms is much smaller than the difference in the atomic number between Ga and $\mathrm{N}$.

To determine the absolute polarity of columnar IDs, we compared numerical simulations of CBED patterns with experimental patterns. Fig. 3d shows a simulated pattern corresponding to that measured for the ID shown in Fig. 3c. This comparison demonstrates that IDs have Al-polarity and that they are embedded in a matrix having N-growth polarity. To assure correctness of the polarity assignment we checked this method for a standard sample i.e. a GaN layer grown with Ga-polarity.

Having demonstrated the existence of IDs in MOCVD AlN/sapphire, we now compare them with IDs in GaN films on sapphire. The latter have been studied extensively in recent years $[6,8-12]$. Several configurations of IDs have been reported for GaN/sapphire, and they seem to correlate with growth methods. "House" shaped domains have been reported for GaN on sapphire films grown by MOCVD [6,8]; they have also been reported for some films grown by hydride vapour phase epitaxy (HVPE) [8] and have been shown to have Npolarity [13]. Because GaN having N-polarity grows slower than GaN having Ga-polarity $[14,15]$, "house" shaped domains are overgrown by the matrix material and are localized near the GaN/sapphire interface.

Two configurations of IDs, reported for GaN/sapphire grown by molecular beam epitaxy (MBE), appear to correlate with two distinct surface morphologies [9-11]. In both configurations, IDs consist of straight, narrow columns extending from the interface to the film surface. Their boundaries are on $\{1 \underline{100}\}$ planes. The first configuration, which is found 
in layers that have a flat surface morphology, consists of very narrow domains, with an average diameter of about $10 \mathrm{~nm}$ [9]. The second ID configuration was found in layers having small pyramids on the surface that were about $100 \mathrm{~nm}$ wide and $75 \mathrm{~nm}$ high. The IDs were up to several tens of nanometers wide [10]; they emerged at the film surface in the centers of the pyramids. It has been determined that these defects were Ga-polarity domains that grow within an N-polarity matrix, and it has been proposed that the pyramids formed on the surface because of the higher growth rate of these domains.

IDs present in our MOCVD AIN/sapphire film are similar to the IDs, just described, within N-polar MBE-grown GaN/sapphire films. In both, $\mathrm{AlN}$ and $\mathrm{GaN}$, inversion domains nucleate at the interface with sapphire and propagate through the entire film; having a higher growth rate, they emerge at the surface in the centers of the pyramids composed of opposite polarity. Moreover, the IDs have group III metal-polarity and are embedded in an N-polarity matrix. However, IDBs in AlN, unlike IDBs in GaN, form a small angle with the c-axis. This may suggest that there is a preferential growth on such slightly inclined planes.

There is a further similarity between IDs in AlN and GaN. For GaN it has been shown that Ga-polarity material grows faster but that its etch rate is very slow compared to that of N-polarity GaN [16,17]. For AlN we have just shown that IDs, which have Al-polarity, grow faster than the N-polarity matrix. Therefore, following the analogy to $\mathrm{GaN}$, one expects these IDs to etch much more slowly than the matrix. To test this hypothesis we etched a TEM specimen for $30 \mathrm{~s}$ in a $50 \%$ water solution of $\mathrm{KOH}$ at $80^{\circ} \mathrm{C}$ and then examined the sample using TEM. Etching changed the morphology of the AlN layer significantly, as can be seen in Fig. 1d. The layer lost its continuity, and separated pyramids (islands) could be seen. Each pyramid contained a columnar ID at its center. The average 
height of these pyramids is almost equal to the thickness of the initial layer, suggesting that pyramid tips, which contained IDs, were scarcely affected by the etching. Moreover, we observed several pyramids that contained "naked" segments of columnar IDs protruding from the apices of the pyramids. This clearly shows that for AlN the etch rates of areas having Al-polarity is much slower compared to the etch rates of areas that grew with $\mathrm{N}$ polarity.

In summary, V-like columnar defects in MOCVD-grown AlN/sapphire correlate with small islands on the surface that are observed by AFM. TEM studies proved that these defects are IDs. They resemble IDs reported for MBE-grown GaN layers with N-polarity grown on sapphire. However, it is not clear yet why ID boundaries in AlN films grow at $2 \pm$ $0.5^{\circ}$ off the c-axis. Our results indicate that these V-like IDs have Al-polarity. They and grow faster and etch more slowly than the N-polarity matrix. Further studies are in progress to propose a model for their IDBs.

Work at LBNL was supported in part by AFOSR No. FQ86710200852, through the U.S. DoE under Contract No. DE-AC03-76SF0098. The TEM group (J.J. and Z.L.-W.) would like to acknowledge the use of the facilities at the National Center for Electron Microscopy at LBNL. Work at AFRL/SNH was supported by AFOSR. The authors thank Dr. Michael N. Alexander for fostering and encouraging the LBNL-AFRL collaboration, and for his close reading of the manuscript. 


\section{References}

1. GaN and Related Materials for Devices Applications, in MRS Bull. 22 (1997).

2. L. F. Eastman, U. K. Mishra, IEEE Spectrum, May 2002, pp. 29-33.

3. T. Shiosaki, T. Yamamoto, T. Oda and A. Kawabada, Appl. Phys. Lett. 36 6431(1980).

4. Qing Paduano and David Weyburne, Jpn. J. Appl. Phys. 42, 1590 (2003).

5. A. D. Westwood, M. R. Notis, J. Am. Ceram. Soc. 74, 1226 (1991).

6. X. H. Wu, L. M. Brown, D. Kapolnek, S. Keller, B. Keller, S. P. DenBaars, and J. S. Speck, J. Appl. Phys. 80, 3228 (1996).

7. R. Serneels, M. Snykers, P. Delavignette, R. Gevers, and S. Amelinckx, Phys. Status Solidi B 58, 277(1973).

8. L. Romano, J. Northrup, and M. O'Keefe, Appl. Phys. Lett. 69, 2394 (1996).

9. V. Potin, P. Ruterana, and G. Nouet, J. Appl. Phys. 82, 2176 (1997).

10. V. Potin, P. Ruterana and G. Nouet, Mater. Sci. \& Eng. B 59, 173 (1999).

11. H. Zhou, F. Phillipp, M. Gross, H. Schroder, Mater. Sci. \& Eng. B 68, 26 (1999).

12. D. Cherns D, W. T. Young, F. A. Ponce, Mater. Sci. \& Eng. B 50, 76 (1997).

13. J. Jasinski and Z. Liliental-Weber, J. Electron. Mat. 31, 429 (2002).

14. B. Daudin, J. L. Rouviere, and M. Arlery, Appl. Phys. Lett. 69, 2480 (1996).

15. E. C. Piquette, P. M. Bridger, Z. Z. Bandic, T. C. McGill TC. J. Vac. Sci. \& Tech. B, 17, 1241 (1999).

16. J. L. Weyher, P. D. Brown, J. L. Rouvière, T. Wosinski, A. R. A. Zauner and I. Grzegory, J. Cryst. Growth 210, 151 (2000).

17. F. Yun, D. Huang, M. A. Reshchikov, T. King, A. A. Baski, W. Litton, J. Jasinski, Z. Liliental-Weber, and H. Morkoç, Phys. Status Solidi B 228, 543 (2001). 


\section{Figure Captions}

Fig. 1. (a), (b) AFM images of the AlN layer surface, showing the surface potential and the height change, respectively. The grey scale in (b) ranges from 0 (the darkest regions) to 50 $\mathrm{nm}$ (the brightest regions). Notice a number of small islands. (c) Bright-field multi-beam cross-sectional TEM micrograph of the AIN layer grown on sapphire. Notice a number of Vlike columnar defects. The well-faceted tip of the defect is shown clearly in the inset. (d) Bright-field TEM image of the AlN layer after wet etching in a hot aqueous $\mathrm{KOH}$ solution. Notice the well-defined pyramids that are present where, before etching, there was a continuous film. Two partially “naked” IDs are indicated by arrows.

Fig. 2. Dark-field TEM images of the AIN layer recorded with a g-vector (a) perpendicular and (b) parallel to the c-axis.

Fig. 3. (a), (b) Dark-field TEM images of the AlN layer recorded with g-vectors (0002) and (0002), respectively. Notice the contrast inversion between regions of inverted polarity. (c) CBED [1 100$]$ patterns recorded at the matrix and the ID regions indicated by white circles on the cross-sectional TEM image. Notice that the ID pattern is reversed compared to that of the matrix (small arrows show the location of characteristic dark bends in these patterns). (d) CBED [1100] pattern calculated for a $68 \mathrm{~nm}$ thick AlN sample with Al-polarity (schematic arrangement of atoms along the c-axis is shown on the right side). 

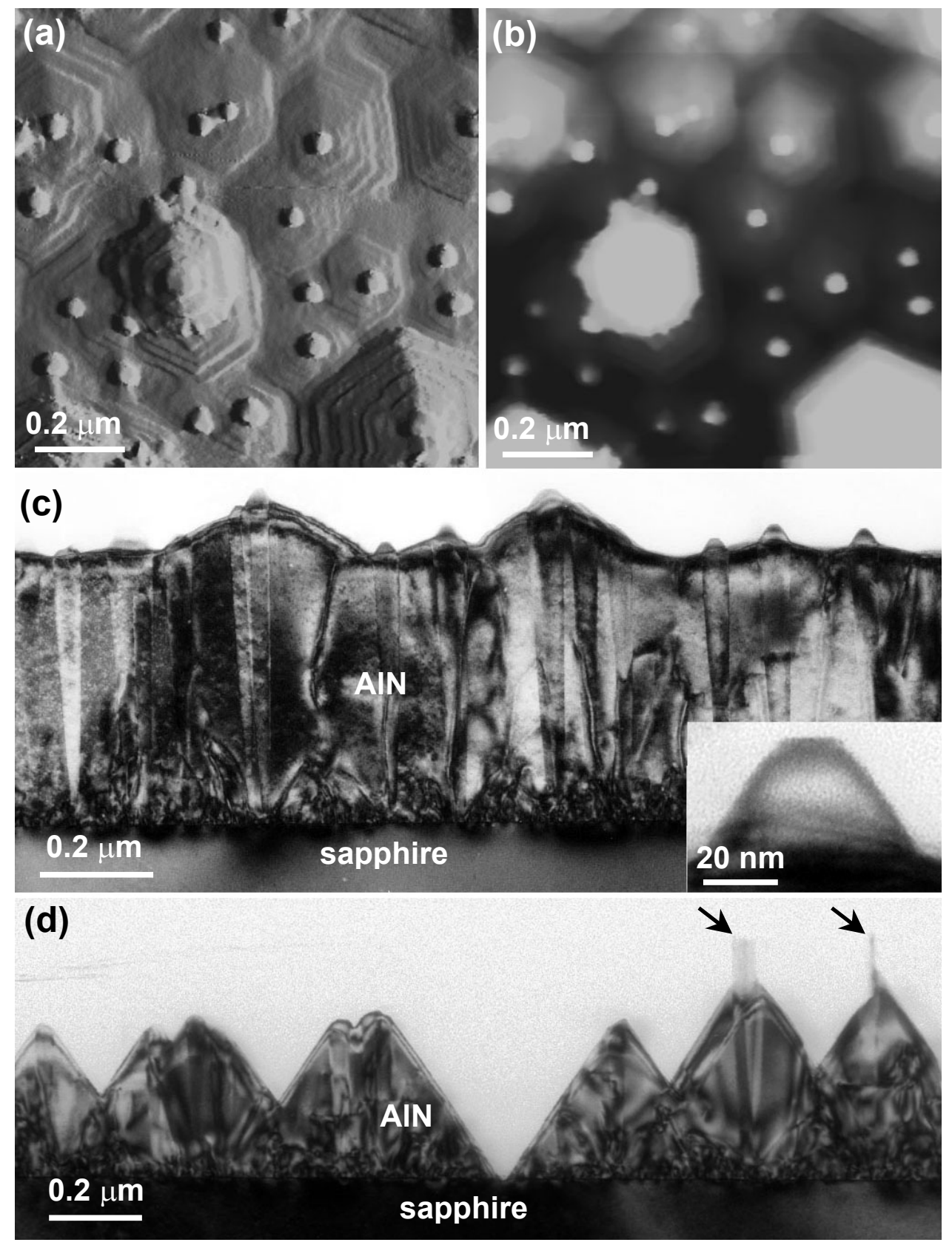

J. Jasinski et al., Fig. 1 

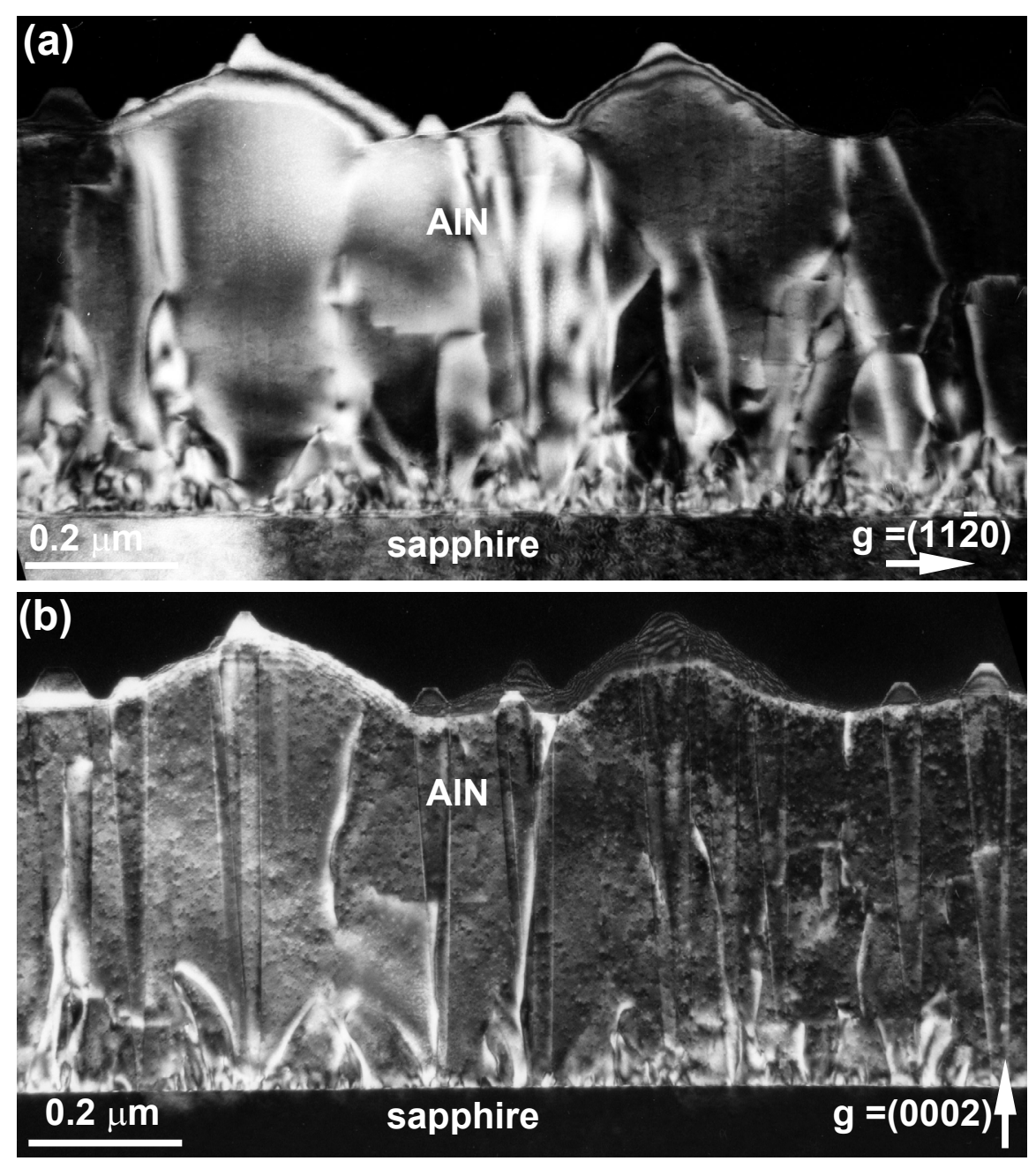

J. Jasinski et al., Fig 2 


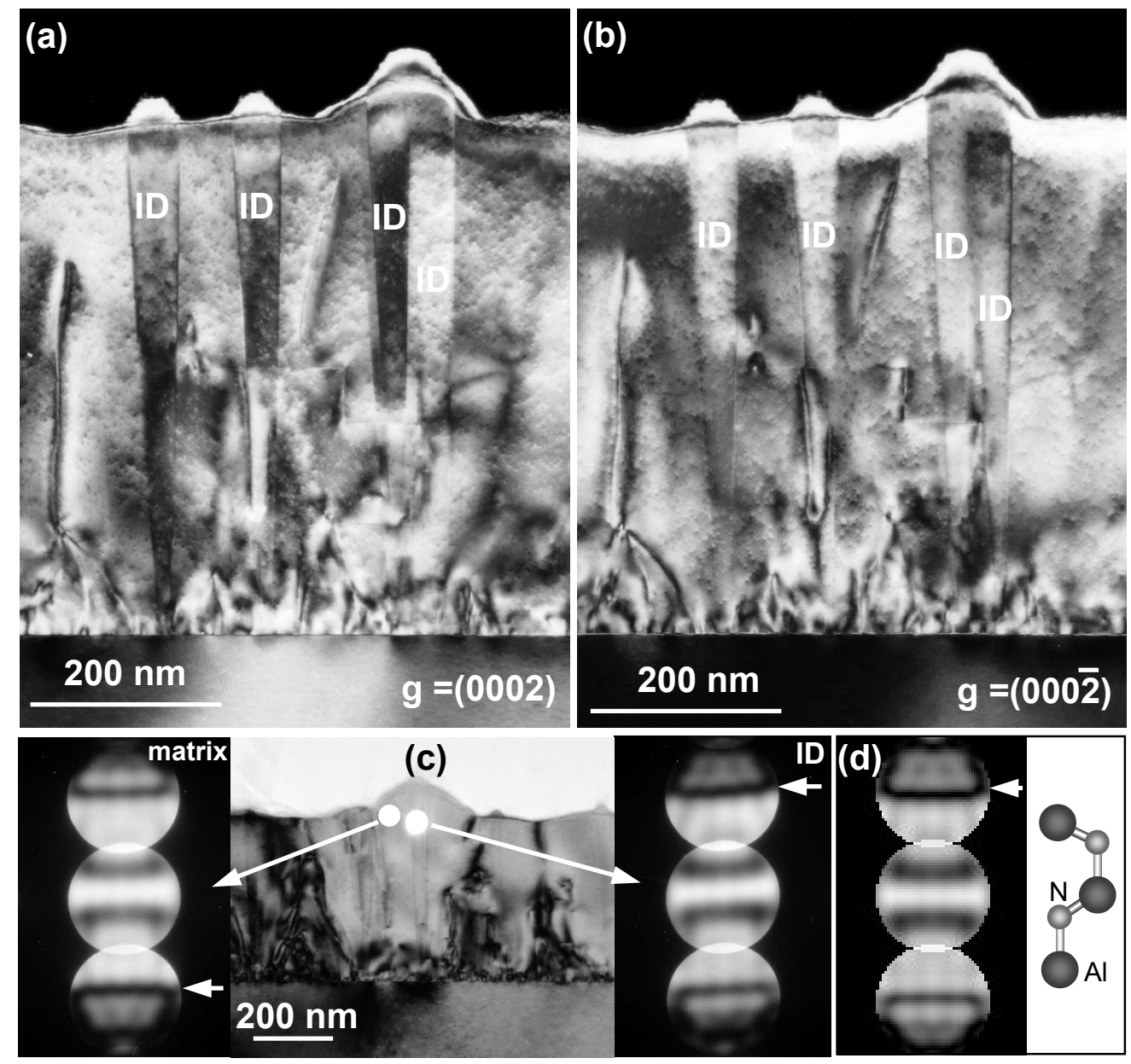

J. Jasinski et al., Fig. 3 